\title{
Mitochondrial Respiration Scavenges Extramitochondrial Superoxide Anion Via a Nonenzymatic Mechanism
}

\author{
David M. Guidot, John E. Repine, Andrew D. Kitlowski, Sonia C. Flores, Sally K. Nelson, Richard M. Wright, \\ and Joe M. McCord \\ Webb-Waring Institute for Biomedical Research and the University of Colorado Health Sciences Center, Denver, Colorado 80262
}

\begin{abstract}
We determined that mitochondrial respiration reduced cytosolic oxidant stress in vivo and scavenged extramitochondrial superoxide anion $\left(\mathrm{O}_{i}^{-}\right)$in vitro. First, Saccharomyces cerevisiae deficient in both the cytosolic antioxidant cuprozinc superoxide dismutase ( $\mathrm{Cu}, \mathrm{Zn}$-SOD) and electron transport $\left(\mathrm{Rho}^{\circ}\right.$ state) grew poorly $(P<0.05)$ in $21 \% \mathrm{O}_{2}$ compared with parent yeast and yeast deficient only in electron transport or $\mathrm{Cu}, \mathrm{Zn}-\mathrm{SOD}$, whereas anaerobic growth was the same $(P>0.05)$ in all yeast. Second, isolated yeast and mammalian mitochondria scavenged extramitochondrial $\mathrm{O}_{i}^{\bar{z}}$ generated by xanthine/xanthine oxidase. Yeast mitochondria scavenged $42 \%$ more $(P<0.05)$ extramitochondrial $O_{\bar{i}}$ during pyruvate/malate-induced respiration than in the resting state. Addition of either antimycin (respiratory chain inhibitor) or FCCP (respiratory chain uncoupler) prevented increased $\mathrm{O}_{\bar{i}}^{\bar{i}}$ scavenging. Mitochondria isolated from yeast deficient in the mitochondrial manganous superoxide dismutase $(\mathrm{Mn}-\mathrm{SOD})$ increased $(P<0.05) \mathrm{O}_{i}^{\overline{2}}$ scavenging $56 \%$ during respiration. This apparent SOD activity, expressed in units of SOD activity per milligram of mitochondrial protein, was the same $(9 \pm 0.6$ vs. $10 \pm 1.0 ; P$ $=0.43$ ) as the $\mathrm{O}_{i}^{-}$scavenging of mitochondria with MnSOD, suggesting that respiration-dependent mitochondrial $\mathrm{O}_{\overline{2}}^{\overline{-}}$ scavenging was nonenzymatic. Finally, isolated rat liver and lung mitochondria also increased $(P<0.05) \mathrm{O}_{i}^{-}$scavenging during respiration. We speculate that respiring mitochondria, via the protonmotive pump, present a polarized, proton-rich surface that enhances nonenzymatic dismutation of extramitochondrial $\mathrm{O}_{\bar{i}}^{\overline{2}}$ and that this is a previously unrecognized function of mitochondrial respiration with potential physiological ramifications. (J. Clin. Invest. 1995. 96:1131-1136.) Key words: superoxide dismutase $\bullet$ xanthine oxidase $\cdot$ lung $\cdot$ liver $\cdot$ yeast
\end{abstract}

\section{Introduction}

The superoxide anion $\left(\mathrm{O}_{\overline{2}}^{\overline{2}}\right)$ has attracted intense scientific scrutiny since the discovery of superoxide dismutase (SOD) (1)

Address correspondence to David M. Guidot, M.D., Medical Service (111), Atlanta VA Medical Center, 1670 Clairmont Road, Decatur, GA 30033. Phone: 404-728-7730; FAX: 404-728-7750.

Received for publication 6 February 1995 and accepted in revised form 10 May 1995.

The Journal of Clinical Investigation, Inc.

Volume 96, August 1995, 1131-1136 uncovered the ubiquitous presence of this oxygen radical in aerobic organisms. The past quarter century has seen an explosion in our knowledge of how $\mathrm{O}_{\dot{2}}^{\overline{2}}$ and other reactive oxygen species, including hydrogen peroxide $\left(\mathrm{H}_{2} \mathrm{O}_{2}\right)$ and the hydroxyl radical $\left({ }^{\circ} \mathrm{OH}\right)$, are formed and react in biological systems (2). Reactive oxygen species are now known to play useful roles, such as bacterial killing by neutrophils and macrophages, as well as to contribute to pathologic states such as ischemiareperfusion injury, radiation damage, and aging. In the normal state, complicated systems tightly control the intracellular levels of reactive oxygen species and maintain a delicate oxidantantioxidant balance. However, any disruption in this balance can lead to significant cellular damage via oxidation of membrane lipids, DNA, and enzymes.

The intracellular sources of $\mathrm{O}_{\overline{2}}^{\bar{z}}$ generation have also attracted attention. Mitochondria, which account for $>90 \%$ of the oxygen consumption in most aerobic cells, generate significant amounts of $\mathrm{O}_{2}^{-}$and $\mathrm{H}_{2} \mathrm{O}_{2}$ in vitro (3). Recently, we constructed Saccharomyces cerevisiae strains deficient in Mn-SOD with and without specific concomitant disruptions in electron transport and determined that mitochondrial respiration is a significant source of $\mathrm{O}_{\bar{i}}^{-}$in hyperoxia in vivo (4). To test the hypothesis that vulnerable targets of mitochondrial $\mathrm{O}_{\overline{2}}^{\overline{2}}$ included cytosolic molecules, we constructed yeast deficient in $\mathrm{Cu}, \mathrm{Zn}$-SOD with and without concomitant disruptions in electron transport. We predicted that disrupting electron transport would decrease oxygen sensitivity in $\mathrm{Cu}, \mathrm{Zn}$-SOD-deficient yeast. To our surprise, loss of electron transport increased oxygen sensitivity in $\mathrm{Cu}, \mathrm{Zn}$ SOD-deficient yeast. To examine this apparent paradox, we isolated mitochondria from yeast and tested their ability to scavenge extramitochondrial $\mathrm{O}_{\dot{2}}^{\overline{2}}$. We then extended these studies to isolated yeast mitochondria deficient in Mn-SOD as well as to isolated mammalian mitochondria.

\section{Methods}

Construction of yeast deficient in $\mathrm{Cu}, \mathrm{Zn}$-SOD. A plasmid containing a deletion construct of the $S$. cerevisiae sod1 gene subcloned into pUC19 was generously provided by Dr. Edith Gralla and Dr. Joan Valentine at UCLA. The sod1 region contained a $0.6-\mathrm{kb}$ deletion in the coding region. The $S$. cerevisiae gene encoding TRP1 was subcloned into the sod1 region at a central AsuII site. The recombinant sod1 disruption carrying the TRP1 insertion was then linearized, isolated, and used to transform S. cerevisiae strains JM43 and JM43Rho ${ }^{\circ}$ (Table I). Transformed yeast which grew on tryptophan-deficient minimal medium agar were screened for the sod1 disruption by protein activity gel electrophoresis (5). The resultant yeast strains were each selectively deficient in $\mathrm{Cu}, \mathrm{Zn}$ SOD (Table I). DGY4 was derived from JM43 and had normal electron transport. DGY5 was derived from JM43Rho ${ }^{\circ}$ and was completely deficient in electron transport. 
Table I. Legend for Yeast Strains Used in This Study

\begin{tabular}{lllc}
\hline Yeast strain & \multicolumn{1}{c}{ SOD activity } & Electron transport & Reference \\
\hline JM43 & Complete & Complete & 4 \\
JM43Rho $^{\circ}$ & Complete & Absent & 4 \\
DGY1 & Mn-SOD deficient & Complete & 4 \\
DGY4 & $\mathrm{Cu}$, Zn-SOD deficient & Complete & This study \\
DGY5 & $\mathrm{Cu}$, Zn-SOD deficient & Absent & This study \\
\hline
\end{tabular}

Yeast growth conditions. Yeast were grown in an environmental shaker (Labline, Melrose Park, IL) at $28^{\circ} \mathrm{C}$ in either $21 \% \mathrm{O}_{2}$ (room air in Denver, altitude 5,280 feet) or anoxia $\left(95 \% \mathrm{~N}_{2} / 5 \% \mathrm{CO}_{2}\right.$ bubbled continuously into the medium) in standard Yeast extract-Peptone-Dextrose liquid medium, composed of $1 \%$ Bacto Yeast Extract (Difco Laboratories Inc., Detroit, MI), 2\% Bacto-Peptone (Difco Laboratories Inc.), and $2 \%$ dextrose.

Assessment of yeast growth. Growth of yeast was determined by sequentially assessing optical density (OD) of each culture at defined time points using a standard spectrophotometer (Klett-Summerson, New York). Increasing density, reflective of cell growth, was expressed as the change in OD ( $\Delta$ Klett units).

Yeast mitochondria isolation. The parent strain JM43 and the MnSOD-deficient strain DGY1 were used in these experiments. Yeast were grown to mid-log phase in liquid medium containing $2 \%$ lactic acid, $0.3 \%$ Bacto Yeast Extract (Difco Laboratories Inc.), $\mathrm{CaCl}_{2}(0.5$ grams/liter), $\mathrm{NaCl}$ (0.5 grams/liter), $\mathrm{MgCl}_{2}$ (0.6 grams/liter), $\mathrm{NH}_{4} \mathrm{Cl}$ ( 1 gram/liter), $\mathrm{KH}_{2} \mathrm{PO}_{4}$ ( $1 \mathrm{gram} /$ liter), and $\mathrm{pH}$ adjusted to 5.5 with $\mathrm{NaOH}$. Yeast were harvested at $4,000 \mathrm{~g}$ for $5 \mathrm{~min}$, resuspended at 0.2 grams $/ \mathrm{ml}$ in $0.1 \mathrm{M}$ Tris base containing $2.5 \mathrm{mM}$ dithioerythreitol (Sigma Immunochemicals, St. Louis, MO), and incubated for $30 \mathrm{~min}$ at $28^{\circ} \mathrm{C}$. Yeast were then washed twice with water and resuspended at 0.25 grams $/ \mathrm{ml}$ in buffer containing $1.35 \mathrm{M}$ sorbitol and $50 \mathrm{mM}$ sodium citrate at $\mathrm{pH}$ 5.8. Yeast were then incubated for $1-2 \mathrm{~h}$ with Zymolyase$20 \mathrm{~T}$ (10 mg/gram of cells, ICN Biochemicals Inc., Costa Mesa, CA). Spheroplasts were then gently washed twice in buffer containing 1.35 $M$ sorbitol and $2 \mathrm{mM}$ EDTA, pelleted at $4,000 \mathrm{~g}$, and resuspended in $5 \mathrm{ml}$ of buffer containing $0.6 \mathrm{M}$ mannitol, $2 \mathrm{mM}$ EDTA, $0.5 \%$ fatty acid-free BSA (Sigma Immunochemicals), $1 \%$ Trasylol (Sigma Immunochemicals), $0.5 \mathrm{mM}$ phenylmethylsulfonylfluoride (Sigma Immunochemicals), $0.25 \mathrm{mM} N \alpha$-p-tosyl-L-lysine chloromethyl ketone (Sigma Immunochemicals), and $0.25 \mathrm{mM} \mathrm{N} N$-tosyl-L-phenylalanine chloromethyl ketone (Sigma Immunochemicals). Spheroplasts were homogenized in a tight-fitting Dounce homogenizer, spun at $4,000 \mathrm{~g}$ for 10 $\mathrm{min}$, and the supernatant was separated and spun at 7,500 $\mathrm{g}$ for $15 \mathrm{~min}$ to recover the mitochondrial pellet. Mitochondria were gently resuspended in 100-200 $\mu \mathrm{l}$ of buffer containing $0.6 \mathrm{M}$ mannitol, $20 \mathrm{mM}$ Hepes, $10 \mathrm{mM}$ potassium phosphate ( $\mathrm{pH} 7.4)$, and $2 \mathrm{mM} \mathrm{MgCl}_{2}$. Confirmation of intact mitochondrial isolation was confirmed for each preparation by measuring the respiratory control ratio in an oxygen electrode (Yellow Springs Instrument Co., Yellow Springs, $\mathrm{OH}$ ).

Rat liver and lung mitochondria isolation. Freshly harvested livers and lungs from healthy adult male Sprague-Dawley rats were minced and placed in cold buffer. Mitochondria were then isolated by a standard method (6) and viability was established by measuring the respiratory control ratio of each preparation.

Determination of isolated mitochondria SOD activity. All assays were performed in a $2-\mathrm{ml}$ reaction volume containing $50 \mu \mathrm{M}$ xanthine, $10 \mu \mathrm{M}$ acetylated cytochrome $c(7)$, and catalase $(12 \mathrm{U} / \mathrm{ml}$, Boehringer Mannheim, Mannheim, Germany). In each assay, superoxide anion was generated by adding bovine xanthine oxidase (8). Cytochrome $c$ reduction was determined by measuring absorbance at $550 \mathrm{~nm}(9)$ in a diode array spectrophotometer (Hewlett-Packard Co., Palo Alto, CA). Xanthine oxidase concentration was adjusted to yield a baseline rate of $\sim 0.02$ absorbance/min and that concentration was then used for each

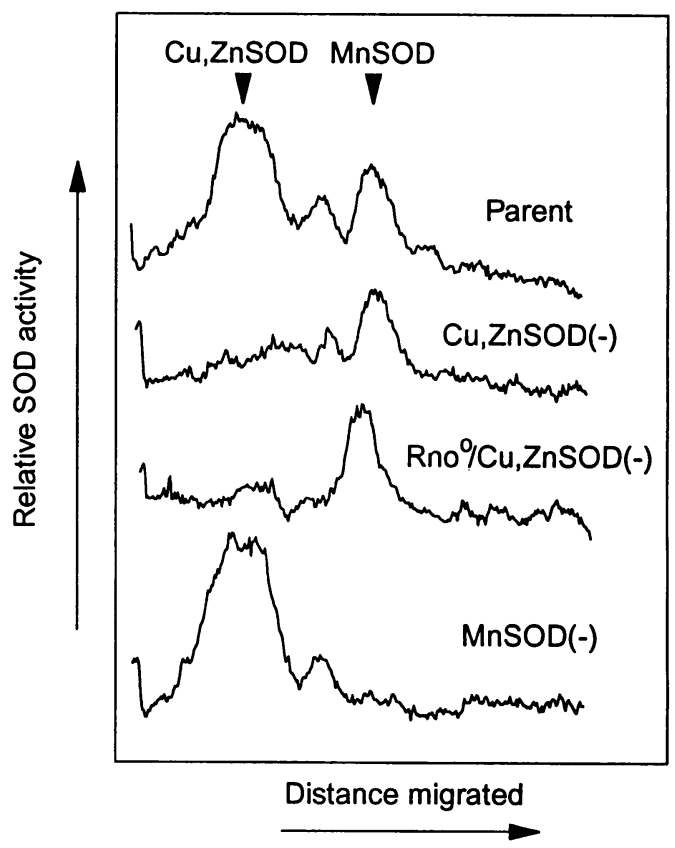

Figure 1. Electropherogram of SOD activity gels (5) of total cellular protein for the parent yeast strain, the $\mathrm{Cu}, \mathrm{Zn}-\mathrm{SOD}(-)$ strain, the Rho $\%$ $\mathrm{Cu}, \mathrm{Zn}-\mathrm{SOD}(-)$ strain, and the Mn-SOD(-) strain (4).

subsequent assay. Baseline SOD activity of freshly isolated yeast, rat liver, and rat lung mitochondria was determined by adding small aliquots $(10-30 \mu \mathrm{l})$ to the reaction mixture and measuring the percent inhibition of cytochrome $c$ reduction (9). The SOD activity of respiring mitochondria was then determined by measuring the SOD of isolated mitochondria given $10 \mathrm{mM}$ pyruvate and $1 \mathrm{mM}$ malate $(10 \mathrm{mM}$ succinate was used with lung mitochondria based on empirical observation that respiration was more efficient with this substrate). In some experiments, antimycin A ( $1 \mu \mathrm{M}$, Sigma Immunochemicals) or carbonyl cyanide $p$-(trifluoromethoxy) phenyl hydrazone (FCCP) ${ }^{1}(0.6 \mathrm{nM}$, Sigma Immunochemicals) was added with the pyruvate and malate. In each condition, SOD activity was calculated and expressed as units per milligram of protein. Protein content of each mitochondrial preparation was determined by the method of Lowry et al. (10).

Statistical methods. For all growth curves, values for OD represent the mean $\pm \mathrm{SE}$ of three or more determinations. Values were compared by one-way ANOVA and corrected by Student-Newman-Keuls test for differences between groups. A $P$ value of $<0.05$ was considered significant. Superoxide scavenging or apparent SOD activity of isolated mitochondrial preparations were compared with the baseline activity for each preparation. Values represent the mean $\pm S E$ of four or more determinations. Values were compared by two-way ANOVA and corrected by Student-Newman-Keuls test for differences between groups. A $P$ value of $<0.05$ was considered significant.

\section{Results}

Confirmation of $\mathrm{Cu}, \mathrm{Zn}$-SOD disruption. Disruption of the $\mathrm{Cu}, \mathrm{Zn}-\mathrm{SOD}$ gene was confirmed by activity gel electrophoresis of total yeast protein. Yeast strains with the $\mathrm{Cu}, \mathrm{Zn}$-SOD disruption had a complete and selective absence of $\mathrm{Cu}, \mathrm{Zn}$-SOD enzyme activity with preservation of Mn-SOD activity (Fig. 1).

1. Abbreviation used in this paper: FCCP, carbonyl cyanide $p$-(trifluoromethoxy) phenyl hydrazone. 


\section{A Nitrogen $\left(0 \% \mathrm{O}_{2}\right)$}

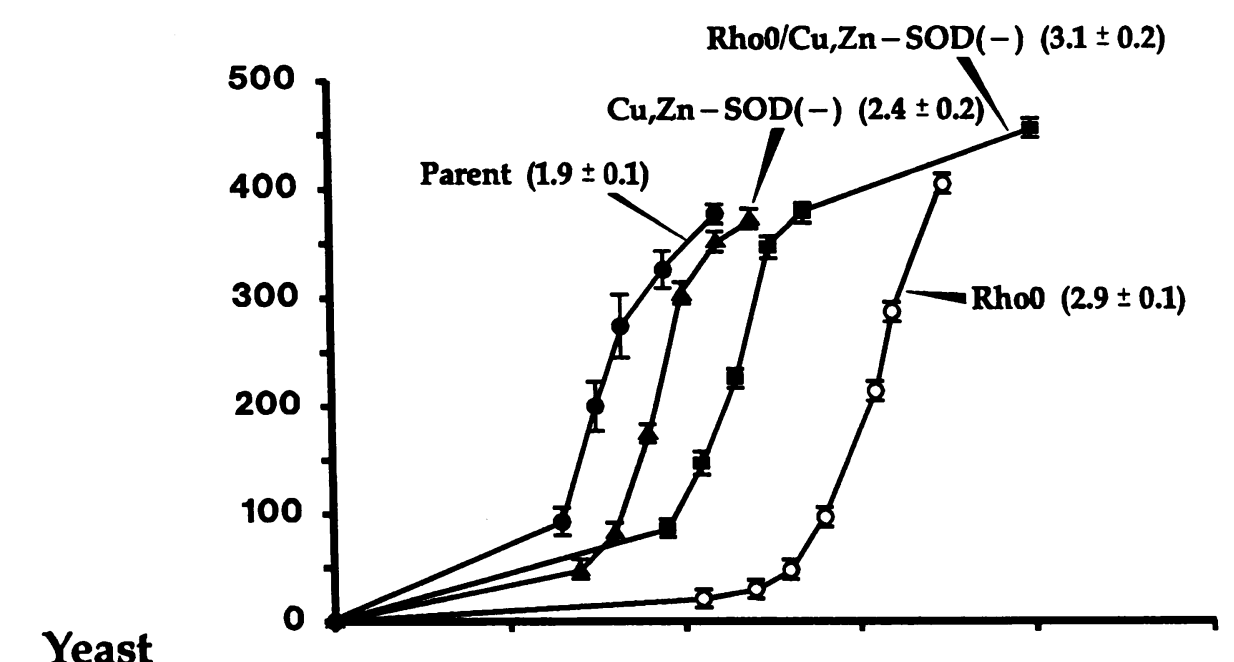

\section{Growth}

(delta Klett)

\section{B Room air $\left(21 \% \mathrm{O}_{2}\right)$}

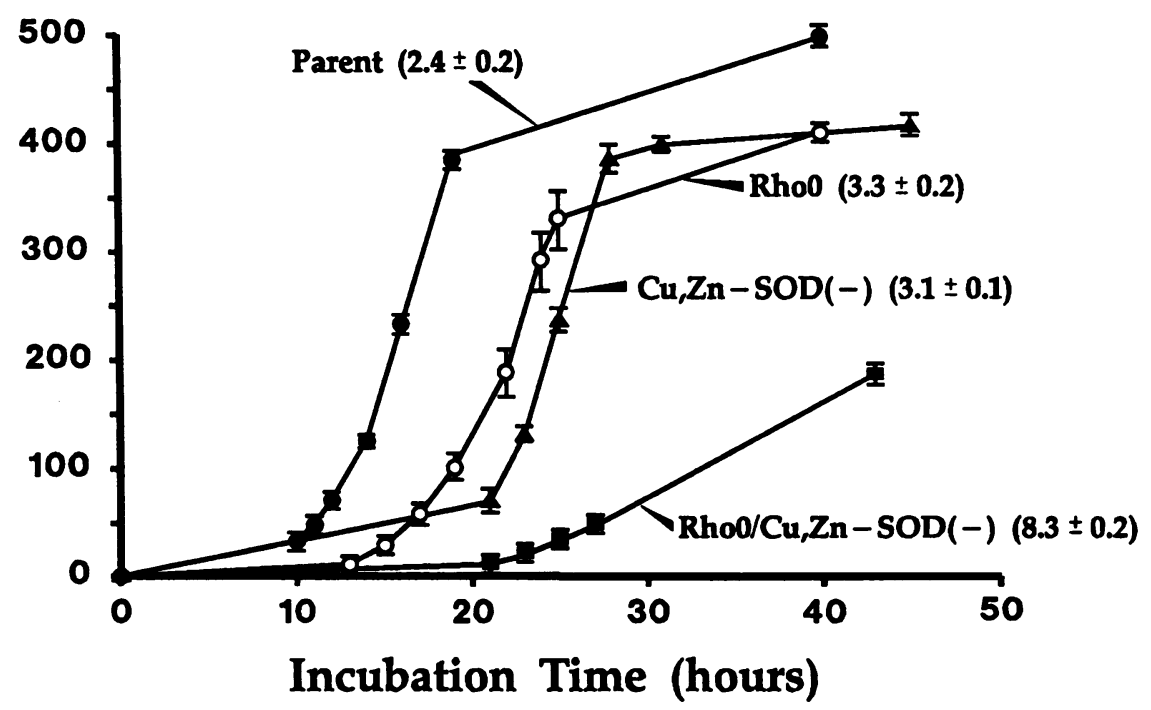

Figure 2. Growth curves in $\mathrm{N}_{2}(A)$ and $21 \% \mathrm{O}_{2}(B)$ for the parent yeast strain, the $\mathrm{Cu}, \mathrm{Zn}-\mathrm{SOD}(-)$ strain, the $\mathrm{Rho}{ }^{\circ}$ strain, and the $\mathrm{Rho} / \mathrm{Cu}, \mathrm{Zn}$ SOD $(-)$ strain. Each value represents the mean \pm SE of four or more determinations. Doubling times (hours) are in parentheses.

The Mn-SOD-deficient strain (4) had preserved $\mathrm{Cu}, \mathrm{Zn}-\mathrm{SOD}$ activity (Fig. 1). The small peaks seen between the two SOD peaks likely represent isolation artifacts, as yeast deficient in both enzymes have no detectable SOD activity (not shown). The Cu,Zn-SOD disruption was also confirmed by Southern blot analysis of genomic DNA from each strain (not shown).

Growth characteristics of $\mathrm{Cu}, \mathrm{Zn}-\mathrm{SOD}$-deficient yeast. Yeast deficient in $\mathrm{Cu}, \mathrm{Zn}-\mathrm{SOD}$ and yeast deficient in both $\mathrm{Cu}, \mathrm{Zn}$ SOD and electron transport had normal growth in nitrogen compared with the respective parent strains JM43 and JM43Rho ${ }^{\circ}$ (Fig. $2 \mathrm{~A}$ ). In contrast, the $\mathrm{Rho}^{\circ} / \mathrm{Cu}, \mathrm{Zn}-\mathrm{SOD}(-)$ strain had significantly impaired growth in normoxia compared with the parent strain, the $\mathrm{Cu}, \mathrm{Zn}-\mathrm{SOD}(-)$ strain, and the JM43Rho ${ }^{\circ}$ strain (Fig. 2 B).

Baseline SOD activity of isolated yeast mitochondria. Iso- lated mitochondria from the parent yeast strain JM43 contained both $\mathrm{Mn}$-SOD and $\mathrm{Cu}, \mathrm{Zn}$-SOD enzyme by activity gel electrophoresis (Fig. 3). By comparison, isolated mitochondria from the Mn-SOD-deficient strain DGY1 had only Cu,Zn-SOD activity (Fig. 3). In both preparations, the source of the $\mathrm{Cu}, \mathrm{Zn}$ SOD enzyme is likely lysosomes and peroxisomes which copurify with mitochondria during isolation (11).

Superoxide scavenging by isolated yeast mitochondria. Mitochondria isolated from the parent strain JM43 had a significant $(P<0.05)$ increase in $\mathrm{O}_{\dot{2}}^{-}$scavenging during respiration induced by pyruvate and malate compared with $\mathrm{O}_{2}^{\overline{-}}$ scavenging by resting mitochondria (Fig. $4 \mathrm{~A}$ ). In contrast, mitochondria given pyruvate and malate in the presence of antimycin $\mathrm{A}$ or FCCP had no significant $(P>0.05)$ increase in $\mathrm{O}_{2}^{\overline{2}}$ scavenging compared with resting mitochondria (Fig. $4 A$ ). In comparison, 


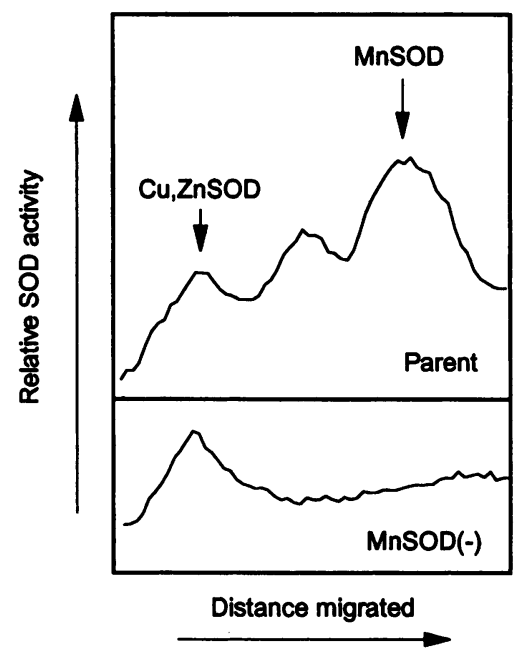

Figure 3. Electropherogram of SOD activity gels (5) of mitochondrial protein for the parent yeast strain and the $\mathrm{Mn}$ SOD $(-)$ strain.

mitochondria isolated from Mn-SOD-deficient yeast also had a significant $(P<0.05)$ increase in $\mathrm{O}_{i}^{\bar{j}}$ scavenging during respiration induced by pyruvate and malate compared with resting mitochondria (Fig. $4 B$ ) but no increase $(P>0.05)$ when given pyruvate and malate in the presence of antimycin $A$ or FCCP (Fig. $4 B$ ).

Superoxide scavenging by isolated rat liver and rat lung mitochondria. Isolated rat liver mitochondria had a significant $(P<0.05)$ increase in $\mathrm{O}_{\overline{2}}^{-}$scavenging during respiration in-

\section{A Yeast mitochondria}

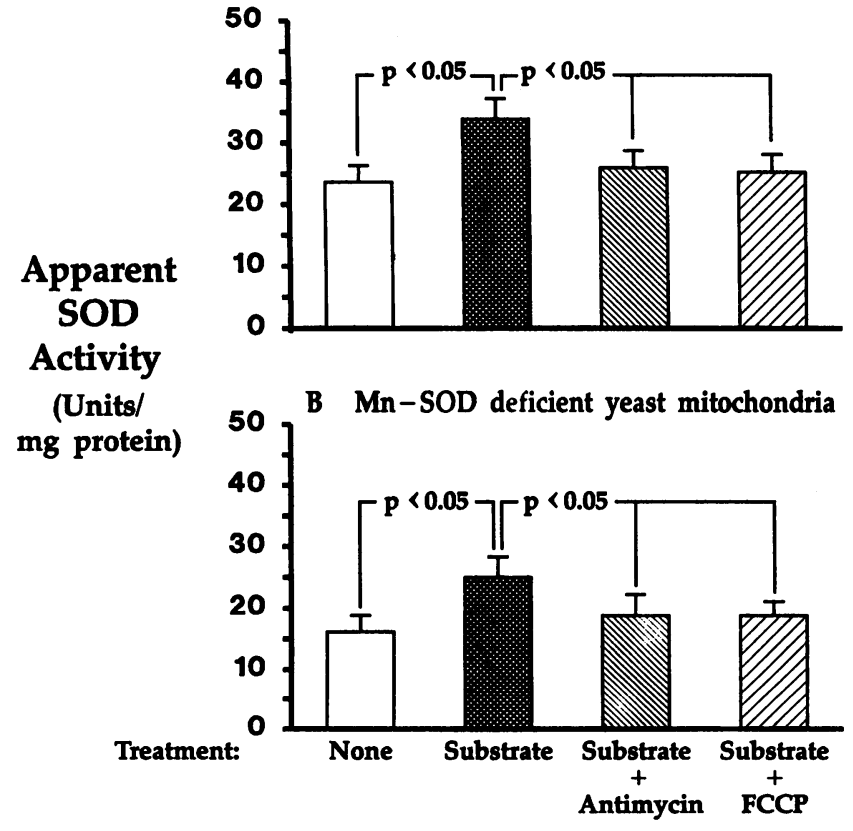

Figure 4. Superoxide scavenging by isolated mitochondria from parent strain $(A)$ and Mn-SOD $(-)$ strain $(B)$ expressed as apparent SOD activity. Activity was determined in the resting state (None), in the presence of pyruvate and malate (Substrate), in the presence of substrate + antimycin, and in the presence of substrate + FCCP. The addition of ADP and inorganic phosphate did not increase $(P>0.05)$ superoxide scavenging by isolated mitochondria in the presence of pyruvate and malate (not shown). Each value represents the mean $\pm S E$ of five or more determinations.

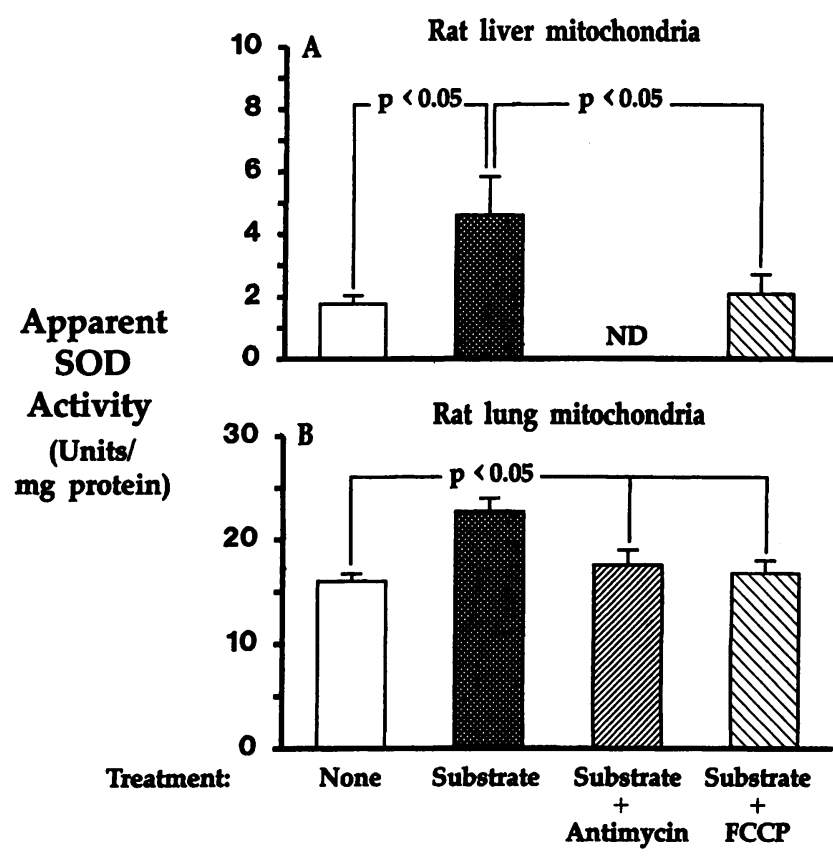

Figure 5. Superoxide scavenging by isolated rat liver mitochondria $(A)$ and rat lung mitochondria $(B)$ expressed as apparent SOD activity. Activity was determined in the resting state (None), in the presence of substrate (pyruvate/malate for liver mitochondria and succinate/malate for lung mitochondria), in the presence of substrate + antimycin (not determined in $A$ ), and in the presence of substrate + FCCP. Each value represents the mean $\pm \mathrm{SE}$ of three or more determinations $(A)$ or seven or more determinations $(B)$.

duced by pyruvate and malate compared with resting mitochondria but no significant $(P>0.05)$ increase when given pyruvate and malate in the presence of FCCP (Fig. $5 A$ ). Isolated rat lung mitochondria also had a significant $(P<0.05)$ respirationdependent increase in $\mathrm{O}_{\dot{i}}^{\bar{z}}$ scavenging prevented by antimycin A or FCCP (Fig. 5 B).

\section{Discussion}

We determined that mitochondrial respiration reduced oxidant stress in yeast in vivo and that mitochondria isolated from yeast and rat liver and rat lung had a respiration-dependent ability to scavenge extramitochondrial superoxide in vitro. These data argue that mitochondria have a previously unrecognized role in cellular antioxidant defense.

Surprisingly, yeast deficient in both $\mathrm{Cu}, \mathrm{Zn}-\mathrm{SOD}$ and electron transport grew poorly in oxygen compared with yeast deficient only in $\mathrm{Cu}, \mathrm{Zn}$-SOD, yeast deficient only in electron transport, or the parent strain. While log phase doubling times were slightly prolonged in oxygen for the parent strain and the single mutants, it was markedly prolonged for the double mutant. As neither defect individually had a substantial effect on growth in oxygen, the oxygen sensitivity in yeast with the combined disruptions was unexpected. Furthermore, as yeast deficient in both electron transport and $\mathrm{Cu}, \mathrm{Zn}-\mathrm{SOD}$ grew normally in nitrogen, the growth inhibition in oxygen could reasonably be ascribed to oxidant stress and not an unidentified metabolic defect caused by the combined genetic disruptions.

This initially paradoxical finding in vivo was supported and 


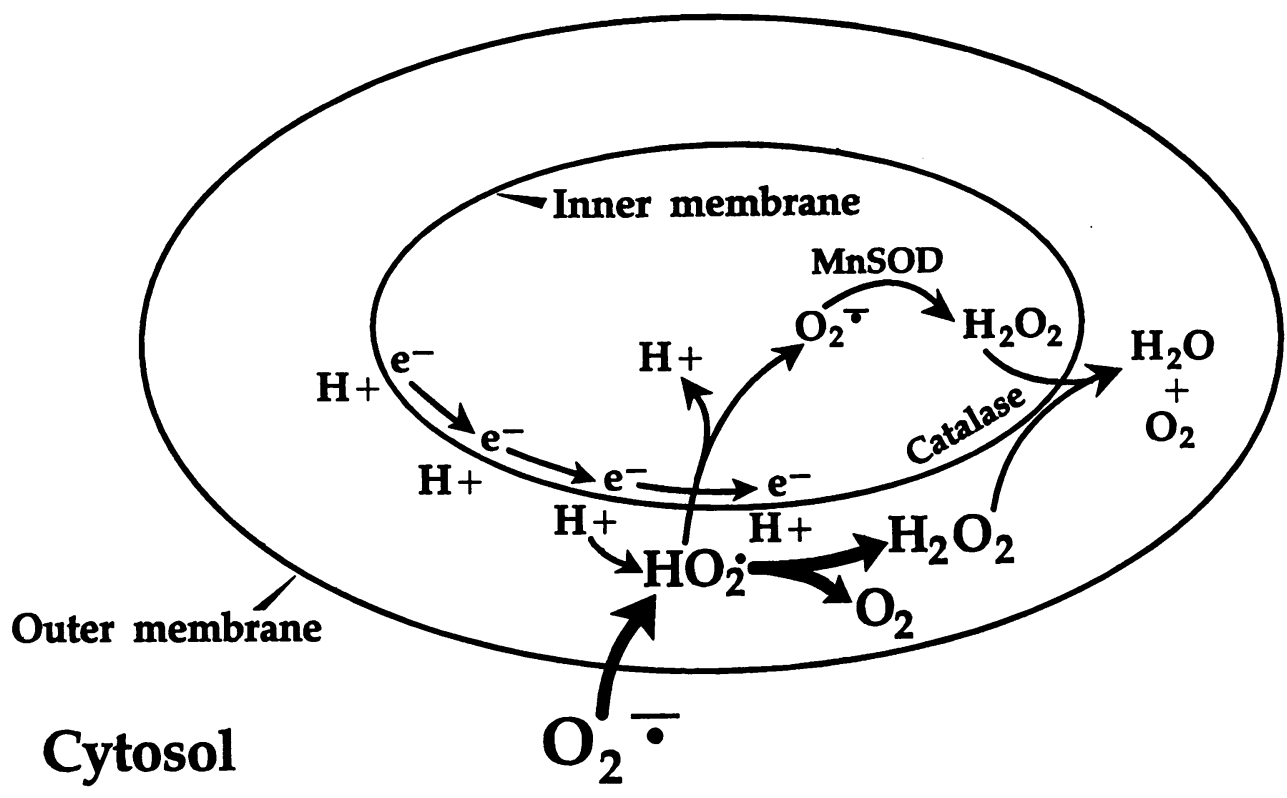

Figure 6. Schematic representation of mitochondrial superoxide scavenging. Cytosolic $\mathrm{O}_{\bar{i}}^{\bar{i}}$ diffusing through the outer mitochondrial membrane will encounter the proton-rich inner membrane of the respiring mitochondria. In this region, spontaneous dismutation of $\mathrm{O}_{2}^{-}$to $\mathrm{H}_{2} \mathrm{O}_{2}$ and $\mathrm{O}_{2}$ is accelerated. Protonated $\mathrm{O}_{\bar{i}}^{-}\left(\mathrm{HO}_{2}^{*}\right)$ could also diffuse across the inner membrane and undergo enzymatic dismutation by Mn-SOD within the matrix. $\mathrm{H}_{2} \mathrm{O}_{2}$ generated by either mechanism can be detoxified by catalase or other peroxidases within the mitochondria. The net $\mathrm{O}_{\overline{2}}^{\bar{i}}$ consumption creates a gradient for $\mathrm{O}_{\overline{2}}^{-}$which favors diffusion from the cytosolic to the mitochondrial space.

explained by the isolated mitochondrial studies. Both yeast and mammalian mitochondria had a significant increase in superoxide scavenging during respiration compared with the resting state. Antimycin A, which inhibits electron transport and thus prevents proton gradient generation, and FCCP, which increases electron transport but dissipates the proton gradient, both eliminated this increase. Furthermore, the addition of ADP and inorganic phosphate, which increase oxygen consumption and electron transport but do not increase the transmembrane potential, did not increase respiration-dependent scavenging by yeast mitochondria. These data argue that superoxide scavenging requires a polarized inner mitochondrial membrane and not electron transport per se. There is precedent for superoxide anion being attracted to a polarized region. The surface of the $\mathrm{Cu}, \mathrm{Zn}$ SOD molecule has a charge distribution that is proposed to guide superoxide anion to the catalytic site (12).

Perhaps even more importantly, respiration-dependent superoxide scavenging did not require Mn-SOD. Mitochondria isolated from yeast which lacked Mn-SOD had the same respiration-dependent capacity to scavenge superoxide as mitochondria with $\mathrm{Mn}-\mathrm{SOD}(9 \pm 0.6$ vs. $10.1 \pm 1.0 \mathrm{U} / \mathrm{mg}$ protein; $P=$ 0.43 ). The increased scavenging induced by pyruvate and malate is inconsistent with an enzymatic mechanism (such as a previously unidentified SOD) and argues for a nonenzymatic mechanism. We speculate that the inner mitochondrial membrane, which becomes positively charged during respiration as protons are translocated to its outer surface, enhances the spontaneous dismutation of extramitochondrial $\mathrm{O}_{\dot{i}}^{-}$which diffuses into the intermembrane space by presenting a localized protonrich (acidic) environment. The second order rate constant for the spontaneous dismutation of superoxide is $\sim 10^{8} \mathrm{M}^{-1} \mathrm{~s}^{-1}$ at $\mathrm{pH} 4.8$ but is more than two orders of magnitude slower at cytosolic $\mathrm{pH}$ (13). As $\mathrm{O}_{\bar{i}}$ rapidly dismutes, a concentration gradient of $\mathrm{O}_{i}^{\overline{1}}$ between the cytosol and the inner membrane is created which would draw in $\mathrm{O}_{2}^{-}$in a manner analogous to how $\mathrm{O}_{2}$ diffuses down the concentration gradient maintained by mitochondrial $\mathrm{O}_{2}$ consumption.

While the primary mechanism of mitochondrial $\mathrm{O}_{\bar{i}}^{\bar{s}}$ scavenging appears to be nonenzymatic, enhanced enzymatic dismu- tation may occur in vivo. At the inner mitochondrial membrane, $\mathrm{O}_{i}^{-}$will protonate to form the uncharged hydroperoxyl radical $\left(\mathrm{HO}_{2}^{*}\right)$ that could diffuse into the mitochondrial matrix (its solubility is similar to $\mathrm{O}_{2}$ ) and could be enzymatically dismuted by Mn-SOD. Dismutation consumes two molecules of $\mathrm{O}_{\dot{i}}^{\overline{1}}$ and yields one molecule of $\mathrm{H}_{2} \mathrm{O}_{2}$, which can also cause oxidative damage. However, $\mathrm{H}_{2} \mathrm{O}_{2}$ is rapidly reduced to $\mathrm{H}_{2} \mathrm{O}$ by catalase and/or other cellular peroxidases. Yeast have a unique cytochrome $c$ peroxidase which scavenges mitochondrial $\mathrm{H}_{2} \mathrm{O}_{2}$ (14), while a specific mitochondrial catalase has been identified in rat heart and kidney mitochondria $(15,16)$. Thus, mitochondria are equipped to reduce $\mathrm{H}_{2} \mathrm{O}_{2}$ generated during $\mathrm{O}_{2}^{-}$scavenging.

Mitochondrial superoxide scavenging capacity in vitro was not equivalent in the different mitochondria examined. Liver mitochondria had the smallest respiration-dependent increase $(2.8 \mathrm{U} / \mathrm{mg}$ protein $)$, with lung intermediate $(6.9 \mathrm{U} / \mathrm{mg}$ protein $)$, and yeast mitochondria the largest capacity $(10 \mathrm{U} / \mathrm{mg}$ protein $)$. While intrinsic differences between yeast and mammalian mitochondria may account for variations in superoxide scavenging, cellular oxidative stress could also explain these differences. Yeast were harvested during log phase growth in lactate broth in a shaking incubator exposed to a $\mathrm{pO}_{2}$ of $\sim 130 \mathrm{mmHg}$, whereas liver cells are relatively quiescent in the healthy animal and exposed to a $\mathrm{pO}_{2}$ of $\sim 40 \mathrm{mmHg}$. Lung mitochondria are exposed to the ambient alveolar $\mathrm{pO}_{2}(\sim 80 \mathrm{mmHg})$. Thus, in this study, mitochondrial superoxide scavenging correlates with the oxygen tension to which the cells were exposed chronically before isolation. An open question at present is whether mitochondrial superoxide scavenging is fixed or responsive to cellular oxidant stress.

These studies reveal a potential role for detoxifying intracellular $\mathrm{O}_{\bar{i}}^{\bar{c}}$ via mitochondrial respiration. Clearly, respiration evolved as a means of coupling electron transfer to ATP formation and accordingly serves as the primary source of energy production in aerobic cells. However, it appears that mitochondrial respiration, by creating a polarized membrane surface, may provide an adventitial function, i.e., scavenging of cytosolic $\mathrm{O}_{\overline{2}}$. Our overall hypothesis is schematically represented in Fig. 6. 
Mitochondrial respiration appears to be intimately involved in the delicate oxidant-antioxidant balance in aerobic cells. This study suggests that mitochondria may provide a heretofore unobserved role in maintaining the cellular redox status by eliminating $\mathrm{O}_{\dot{i}}$ of both mitochondrial and cytosolic origin.

\section{Acknowledgments}

This work was supported by grants from the National Institutes of Health (P50-HL27353 and K-11-HL02690).

\section{References}

1. McCord, J. M., and I. Fridovich. 1969. Superoxide dismutase: an enzymic function for erythrocuprein (hemocuprein). J. Biol. Chem. 244:6049-6055.

2. Cadenas, E. 1989. Biochemistry of oxygen toxicity. Annu. Rev. Biochem. 58:79-110.

3. Cross, A. R., and O. T. G. Jones. 1991. Enzymic mechanisms of superoxide production. Biochim. Biophys. Acta. 1057:281-298.

4. Guidot, D. M., J. M. McCord, R. M. Wright, and J. E. Repine. 1993. Absence of electron transport ( $\mathrm{rho}^{\circ}$ state) restores growth of a manganese-superoxide dismutase-deficient Saccharomyces cerevisiae in hyperoxia: evidence for electron transport as a major source of superoxide generation in vivo. J. Biol. Chem. 268:26699-26703.

5. Salin, M. L., and J. M. McCord. 1974. Superoxide dismutases in polymorphonuclear leukocytes. J. Clin. Invest. 54:1005-1009.

6. Pedersen, P., L. Greenawalt, B. Reynafarje, J. Hullihen, G. Decker, J. Soper, and E. Bustamente. 1978. Preparation and characterization of mitochondria and submitochondrial particles of rat liver and liver-derived tissues. Methods Cell Biol. 20:411-481.

7. Azzi, A., C. Montecucco, and C. Richter. 1975. The use of acetylated ferricytochrome $c$ for the detection of superoxide radicals produced in biological membranes. Biochem. Biophys. Res. Commun. 65:597-603.

8. Waud, W. R., F. O. Brady, R. D. Wiley, and K. V. Rajagopalan. 1975. A new purification procedure for bovine milk xanthine oxidase: effect of proteolysis on the subunit structure. Arch. Biochem. Biophys. 169:695-701.

9. Crapo, J., J. McCord, and I. Fridovich. 1978. Preparation and assay of superoxide dismutases. Methods Enzymol. 53:382-393.

10. Lowry, O. H., N. J. Rosebrough, A. L. Farr, and R. J. Randall. 1951. Protein measurement with the Folin phenol reagent. J. Biol. Chem. 193:265-275. 11. Mertens-Strijthagen, J., C. De Schrijver, S. Wattiaux-De Coninck, and R. Wattiaux. 1979. A centrifugation study of rat-liver mitochondria, lysosomes and peroxisomes during the perinatal period. Eur. J. Biochem. 98:339-352.

12. Getzoff, E. D., J. A. Tainer, P. K. Weiner, P. A. Kollman, J. S. Richardson, and D. C. Richardson. 1983. Electrostatic recognition between superoxide and copper, zinc superoxide dismutase. Nature (Lond.). 306:287-290.

13. Klug, D., J. Rabani, and I. Fridovich. 1972. A direct demonstration of the catalytic action of superoxide dismutase through the use of pulse radiolysis. $J$. Biol. Chem. 247:4839-4842.

14. Yonetani, T. 1965. Studies on cytochrome $c$ peroxidase. II. Stoichiometry between enzyme, $\mathrm{H}_{2} \mathrm{O}_{2}$, and ferrocytochrome $c$ and enzymic determination of extinction coefficients of cytochrome $c$. J. Biol. Chem. 240:4509-4514.

15. Radi, R., S. Sims, A. Cassina, and J. Turrens. 1993. Roles of catalase and cytochrome $c$ in hydroperoxide-dependent lipid peroxidation and chemiluminescence in rat heart and kidney mitochondria. Free Radical Biol. F. Med. 15:653659.

16. Radi, R., J. Turrens, L. Chang, K. Bush, J. Crapo, and B. Freeman. 1991 Detection of catalase in rat heart mitochondria. J. Biol. Chem. 266:22028-22034. 